\title{
PRISM-FILM COUPLING IN ANISOTROPIC PLANAR WAVEGUIDES OF EPITAXIAL (101) RUTILE THIN FIMMS*
}

\author{
C. M. Foster, S.-K. Chan, H.L.M. Chang, \\ R. P. Chiarells, and D. J. Lam \\ Materials Science Division \\ Argonne National Laboratory, Argonne, IL 60439.
}

April 1993

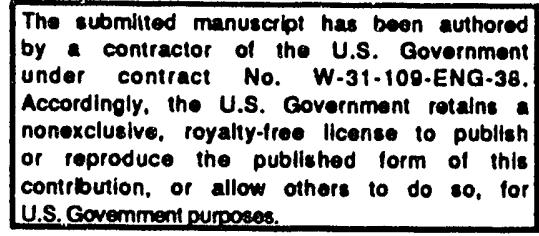

DISCLAIMER

\begin{abstract}
This report was prepared as an account of work sponsored by an agency of the United States Government. Neither the United States Government nor any agency thereof, nor any of their employees, makes any warranty, express or implied, or assumes any legal liability or responsibility for the accuracy, completeness, or usefutness of any information, apparatus, product, or process disclosed, or represents that its use would not infringe privately owned rights. Reference herein to any specific commercial product, process, or service by trade name, trademark, manufacturer, or otherwise does not necessarily constitute or imply its endorsement, recommendation, or favoring by the United States Government or any agency thereof. The views and opinions of authors expressed herein do not necessarily state or reflect those of the United States Government or any agency thereof.
\end{abstract}

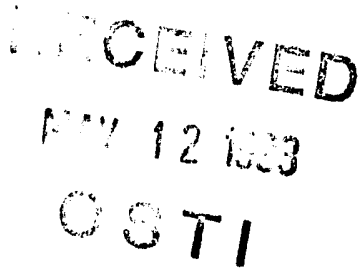

To be presented at the MRS 1993 Spring Meeting, Symposium on "Ferroelectric Thin Films III," San Francisco, CA, April 12-16, 1993, and published in the Proceedings.

\footnotetext{
*Work supported by the U.S. Department of Energy, BES-Materials Sciences, under contract \#W-31-109-ENG-38.
} 


\title{
PRISM-FILM COUPLING IN ANISOTROPIC PLANAR WAVEGUIDES OF EPITAXIAL (101) RUTILE THIN FILMS
}

C. M. Foster, S.-K. Chan, H.L.M. Chang, R. P. Chiarello, and D. J. Lam

Materials Science Division, Argonne National Laboratory, 9700 S. Cass Avenue, Argonne, II 60439

\begin{abstract}
We report optical waveguiding in single-crystal, epitaxial (101) oriented rutile $\left(\mathrm{TiO}_{2}\right)$ thin films grown on $(11 \overline{20})$ sapphire $\left(\alpha-\mathrm{Al}_{2} \mathrm{O}_{3}\right)$ substrates using the MOCVD technique. The propagation constants for asymmetric planar waveguides composed of an anisotropic dielectric media applicable to these films are derived. Modifications to the prism-film coupling theory for this anisotropic case are also discussed. By application of this model to (101) oriented rutile thin films, we directly obtain values of the ordinary and extraordinary refractive indexes, $n_{0}$ and $n_{e}$, of the rutile thin films as well as film thicknesses. We obtain typical values of the refractive indexes $\left(n_{0}=2.5701 \pm 0.0005 ; n_{c}=2.934 \pm 0.001\right)$ near to those for bulk rutile single crystals indicating the exceptional quality of these films.
\end{abstract}

\section{INTRODUCTION}

The tremendous benefits derived from optical communications and the great promise of high-speed optical computers has stimulated interest in the guidance of light by dielectric waveguides. Many aspects of light propagation in waveguides structures, suckt as optical fibers and the thin-film guides of integrated optics, have been investigated in great detail. Moreover, the fundamental propagation characteristics, such as the propagation constants and field distributions of guided modes, have been comprehensively reviewed in the literature [1].

Due to its high refractive index, rutile $\left(\mathrm{TiO}_{2}\right)$ is an ideal material for passive optical waveguides. The reduced defect size and enhanced dielectric properties of single-crystal, epitaxial thin films should significantly reduce optical loss due to grain-boundary scattering in planar waveguides as compared to structures produced from polycrystalline material. Recently, we have studied in detail the crystal structure of rutile thin films on sapphire $(1 \overline{1} \overline{2})$ by high resolution electron microscopy (HREM) and four-circle $x$-ray diffraction [2-3]. These studies have indicated that epitaxial (101) oriented rutile thin films grown on (1120) sapphire substrates by the MOCVD process possess a highly anisotropic single-crystal nature. Consequantly, an understanding of the optical waveguiding in these films must necessarily take into account the anisotropy of the rutile dielectric tensor. For optical waveguide applications, the main parameters of interest which characterize thin films are the ordinary and extraordinary refractive indexes, $n_{0}$ and $n_{e}$, and the film thickness. One method that has been particularly well adapted to this problem in the case of isotropic films, is the prism-coupling technique. This technique was first described by Tien, Ulrich, and Martin [4] and more recently by others [5-6]. Waveguide propagation in arbitrarily oriented, anisotropic, dielectric media has been treated to some degree of generality [7]. The electromagnetic field distributions and propagation constants have been calculated in closed form only in specific cases [9]. However, none of these treatments are directly applicable to (101) oriented rutile films. In this paper, we report optical waveguiding in single-crystal, epitaxial (101) oriented rutile thin films grown on (1120) sapphire substrates using the MOCVD technique. By application of these results, we directly obtain values of the ordinary and extraordinary refractive indexes, $\mathbf{n}_{\mathbf{0}}$ and $\mathbf{n}_{e}$, as well as film thickness for (101) oriented rutile thin films.

\section{THEORY}

We calculate the electromagnetic field distributions and propagation constants for a waveguide by solving Maxwell's equations subject to the appropriate boundary conditions. We endeavor to model a system which is simple enough so that the refractive indexes and film thickness of an anisotropic, (101) oriented orthorhombic thin film can easily be obtained using prism-fil:n waveguide coupling. Therefore, we consider asymmetric planar waveguides composed 
of anisotropic dielectric media, e.g. absorptionless, non-magnetic medium with a dielectric matrix K $[8]$. For this medium, Maxwell's curl equations are

$$
\vec{\nabla} \times \mathbf{E}=-\mu_{0} \dot{\mathbf{H}} \text { and } \vec{\nabla} \times \mathbf{H}=\varepsilon_{0} \mathbf{K} \dot{\mathbf{E}}
$$

where $\dot{\mathbf{E}}$ and $\dot{\mathbf{H}}$ are time derivatives of the fields. Combined, these equations yield

$$
\nabla(\nabla \cdot E)-(\nabla \cdot \nabla) E=-\mu_{0} \varepsilon_{0} K \ddot{E} \text { and } \nabla \times\left[K^{-1} \nabla \times \mathbf{H}\right]=-\mu_{0} \varepsilon_{0} \ddot{\mathbf{H}}
$$

where we use the symbols $e_{0}$ and $\mu_{0}$ for the permittivity and the permeability of free space. Solutions of Eq. (3) or (4) yield either the electric field, E, or the magnetic field, $H$, respectively, and then either Eq. (1) or (2) can be used to obtain the other field.

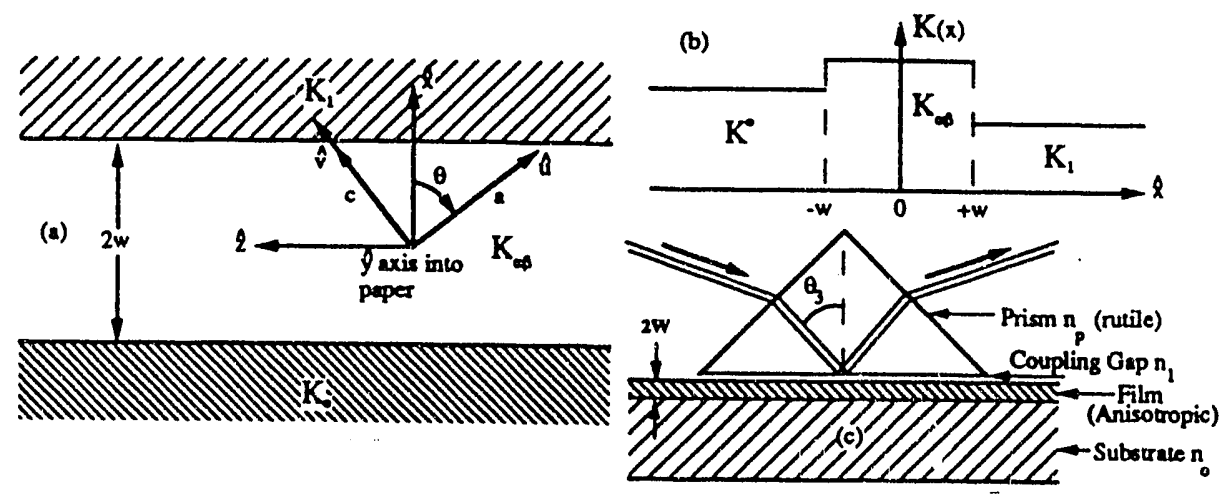

Fig. 1. (a) The coordinate system $(u, y, v)$ in which the dielectric tensor of the rutile film is diagonal, and the coordinate system $(x, y, z)$, which is used to express the spatial dependence of the fields, are shown. (b) The assumed dielectric constant dependence on the coordinate $x$ is shown for an asymmetric nutile thin film. (c) Schematic of a prism-film coupler.

We consider crystalline thin films of at least orthorhombic symmetry with a (101) growth plane. As shown in Fig. 1a, the $\vec{a}$ crystallographic axis of the film is rotated by an angle $\boldsymbol{\theta}$ about the $\vec{b}$-axis with respect to the vector normal to the film surface which we define as the $\overrightarrow{\boldsymbol{x}}$-axis. This angle is determined by $\theta=\tan ^{-1}(a / c)$, where $a$ and $c$ are the unit cell parameters of the material which we will consider to be orthorhombic. This anisotropic medium has a dielectric matrix $K$, thickness $2 w$, and is located between two semi-infinite isotropic media (i. c. air and a substrate). The $\bar{x}^{\prime}$ coordinate system $(u, y, v)$, in which the dielectric tensor of the rutile film is diagonal, is rotated by an angle $\theta$ with respect to the $\ddot{x}$ coordinate system $(x, y, z)$, which is aligned with respect to the dielectric boundaries. It is advantageous to resolve the gradient operator and field vectors along the principal $(u, y, v)$ axes while expressing the spatial dependence of the fields in terms of $(x, y, z)$ coordinates. We will denote components of the diagonal dielectric tensor $K_{\alpha \beta}$ in the $(u, y, v)$ coordinate system as $K_{u}=n_{u}^{2}, K_{y}=n_{y}^{2}, K_{v}=n_{v}^{2}$, and assume that the substrate indexes $K_{0}$ and $K_{1}$ are isotropic.

We search for a solution of Eq. 3 or 4 of the forms $\vec{E}=\vec{e}(x) \exp (i(\omega t-B z))$ and $\overrightarrow{\mathbf{H}}=\overrightarrow{\mathbf{h}}(\mathbf{x}) \exp (\mathbf{i}(\omega t-\beta z))$ corresponding to waves traveling in the $+z$ direction. Here $\omega$ is the angular frequency of the light and $t$ is the time. As shown in Fig. 1b, the dielectric tensors depend only upon the variable $\mathbf{x}$; therefore, $\overline{\mathbf{e}}$ and $\overline{\boldsymbol{h}}$ need only be functions of $\mathbf{x}$. The lack of $\mathbf{y}$ dependence and the diagonal nature of the dielectric tensors in the $(u, y, v)$ coordinate system leads to the decoupling of $e_{y}(x)$ from the $e_{u}(x)$ and $e_{v}(x)$ components of Eq. (3). A similar decoupling occurs for the $h_{y}(x)$ component of Eq. (4). Therefore, transverse electric (TE) and transverse magnetic (TM) modes exist in this case. The explicit forms for the resulting plane wave differential equations for the TE and TM cases are given in Ref. [10]. These differential equations have constant coefficients in the different regions of the waveguide as shown in Fig. la and can be readily solved. For the following analysis, it is convenient to define the following

$$
\mathbf{K}_{\mathbf{x}}^{\prime}=\mathbf{K}_{\mathbf{u}} \mathbf{K}_{\mathbf{v}} / \mathbf{K}_{\mathbf{x}} \text {, }
$$


and

$$
K_{x}=\left\{K_{v} \sin ^{2} \theta+K_{u} \cos ^{2} \theta\right\} ; K_{z}=\left\{K_{u} \sin ^{2} \theta+K_{v} \cos ^{2} \theta\right\}
$$

$$
K_{x z}=\left\{K_{v}-K_{u}\right\} \sin \theta \cos \theta,
$$

where $k_{0}^{2}=\varepsilon_{0} \mu_{0} \omega^{2}$. By application of the usual continuity condition on the electric and magnetic fields at the boundaries, the spatial dependence of the fields can be obtained and are shown in Ref. [10]. For TE modes, the fields depend on the real parameters, $\delta, \gamma, \beta$, and $b$, which are determined by the following four eigenvalue equations

$$
\begin{gathered}
\beta^{2}=x_{0}^{2} K_{0}+\delta^{2} ; \beta^{2}=x_{0}^{2} K_{1}+\gamma^{2} ; \beta^{2}=x_{0}^{2} K_{y}-b^{2} \\
\tan (2 b w)=\frac{(\gamma / b)+(\delta / b)}{1-\left(\gamma \delta / b^{2}\right)} .
\end{gathered}
$$

Explicit forms for $\mathbf{e}(\mathbf{x})$ and $\mathbf{h}(\mathbf{x})$ for the TE modes are given in Ref. [10]. This results from the alignment of the transverse $\mathbf{E}$ field with the principal axis of the dielectric tensor and from the non-magnetic nature of the media. For the TM modes, the fields depend on the real parameters, $\delta$, $\gamma, \beta, b_{1}$ and $b_{2}$, which are determined by Eqs. (14) and (15), and the following

and

$$
b_{1}=\beta K_{x x} / K_{x} ; b_{2}^{2}=K_{0}^{2} K_{x}^{\prime}-\beta^{2}\left\{K_{x}^{\prime} / K_{x}\right\}
$$

$$
\tan \left(2 b_{2} w\right)=\frac{\left(K_{x}^{\prime} \gamma / K_{1} b_{2}\right)+\left(K_{x}^{o} \delta / K_{0} b_{2}\right)}{1-\left(K_{x}^{o} \gamma / K_{1} b_{2}\right)\left(K_{x}^{\prime} \delta / K_{0} b_{2}\right)}
$$

Explicit forms for $\mathbf{e}(x)$ and $h(x)$ for the TM modes are given in Ref. [10]. From these results, if we assume a (101) oriented tetragonal film with properties corresponding to bulk rutile on (1120) sapphire substrate $\left(2 w=0.5332 \mu \mathrm{m}, n_{1}=1.0003, n_{1}=1.766, n_{\text {fim }}^{f}=2.5839, n_{\text {fim }}^{e}=2.8659\right.$ [11] and a laser wavelength of $0.6328 \mu \mathrm{m})$, we find that $\left|h_{\mathbf{x}}(\mathbf{x}) / \mathrm{h}_{\mathbf{z}}(\mathbf{x})\right|<0.01$ for the TE: modes; while $\left|e_{x}(x) / e_{z}(x)\right|<0.01$ for the TM modes. Therefore, the waveguide modes in these films are essentially TEM (transverse electromagnetic) in character. In addition, For the TE0 and TMo modes, we find that the peak intensities inside the film differ by only $11 \%$; while the field intensity at the film-substrate boundary is reduced from the peak intensity by $>3.7 \times 10^{5}$ and $>4.5 \times 10^{6}$ for the zeroth order TE and TM modes, respectively.

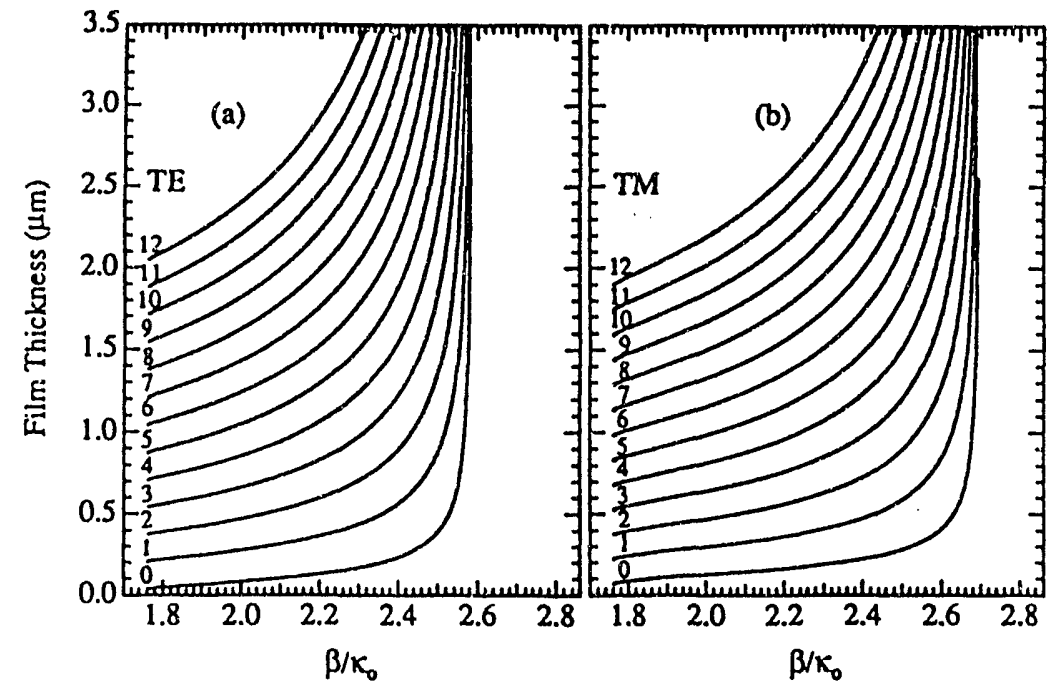

Fig. 2 . The film thickness $2 w$ vs. $\beta / \kappa_{0}$ for the first twelve modes (the mode orders are indicated by number) using $\mathbf{n}_{1}=1.000, \mathbf{n}_{0}=1.758, \mathbf{n}_{\text {film }}^{0}=2.5839, \mathbf{n}_{\text {rilm }}^{\mathrm{e}}=2.8659, \theta=57.215^{\circ}$ and a laser wavelength of $0.6328 \mu \mathrm{m}$; (a) corresponds to TE modes and (b) corresponds to TM modes. 
As shown in the description of the prism-film coupler by Tien a.2d Ulrich [5], efficient waveguide coupling occurs when the component parallel to the film surface of the wave vector of the evanescent fields in the air gap (associated with the light which is totally reflected from the prism base) matches that of one of the characteristic waveguide modes in the film. This component is $\beta=x_{0} n_{p} \sin \theta_{3}$, where $n_{p}$ is the refractive index of the prism and $\theta_{3}$ is the angle with respect to the normal of the light incident upon the prism base (see Fig. 1c). As shown in Ref. [5], propagation will only occur if the total phase shift of all waves which are multiply reflected between the film-substrate and film-air surfaces is equal to a multiple of $2 \pi$. This leads to the expression for the waveguide modes in the very weak coupling limit [5],

$$
2 b w-2 \phi_{01}-2 \phi_{12}=2 m x
$$

where $b$ is the propagation constant, $m$ is the mode order, and $\phi_{01}$ and $\phi_{12}$ are the half-angle reflection phase shifts at the film-substrate and film-air interfaces, respectively. We can generalize Eq. (21) for the case for (101)-oriented crystalline thin films. The expressions for $b$ in this case are given by Eq. (16) for TE modes and Eq. (19) for TM modes. Furthermore, for both TE and TM modes, a single equation for the thickness, $w$, can be obtained by eliminating $\delta, \gamma$, and $\beta$ between the appropriate four eigenvalue equations. From the resulting expressions, the appropriate halfangle phase shifts for TE modes can be extracted [10]. Explicit forms for $\phi_{01}$ and $\phi_{12}$ for both TE and TM modes are given in Ref. [10]. For TE and TM modes, combining Eq. (21) with the appropriate expressions for $\phi_{01}$ and $\phi_{12}$ yield single equations for the film thickness as a function of the propagation constant $\beta$. In Fig. 2, we plot for orders, $m=0$ to 12, the thickness of the film required for a TE mode (a) or a TM mode (b) to propagate with a propagation constant $\beta / k_{0}$ for a tetragonal film with optical properties corresponding to bulk rutile on (1120) sapphire substrate (parameters given above).

\section{RESULTS AND DISCUSSION}

Details of the $x$-ray, Four-circle $x$-ray, and prism-film wave guide coupler have been reported previously [10]. To determine the orientation and the degree of crystallinity of the films quantitatively, we performed $x$-ray scattering measurements, these data are presented else where [3]. The specular and off-specular $x$-ray diffraction spectrum indicated that the film consisted of a single growth plane corresponding closely to the (101) plane of rutile single crystals [3] $\left(2 \theta=36.12^{\circ}\right)$. The rocking curve for the growth plane $(\theta$-scan) indicated an intensity full width at half maximum (FWHM) of $0.30^{\circ}$. The azimuthal $\phi$-scan data detected no in-plane misorientation of the film with respect to the substrate, and showed a FWHM of $0.83^{\circ}$ indicating the very high degree of in-plane epitaxy for this film. A least squares refinement of the 4-circle $x$-ray diffraction results for 13 off-specular reflections was used to determined the lattice parameters for the film of $a=4.59 \AA, b=4.54 \AA$, and $c=2.96 \AA$, with $\alpha=89.8^{\circ}, \beta=88.9^{\circ}$, and $\gamma=89.9^{\circ}$ as compared to bulk tetragonal $\mathrm{TiO}_{2}$ with $\mathrm{a}=\mathrm{b}=4.5937 \AA, c=2.9587 \AA$, and $\alpha=\beta=\gamma=90.0^{\circ}$. Thus, according to the $x$-ray measurements, the film has a highly-crystalline, distorted rutile structure, particularly with respect to the b-axis.

As discussed in Ref. [3], the film-substrate interface along $\mathrm{TiO}_{2}$ [101] direction is sharp at the atomic level and structurally coherent, due to the small lattice mismatch $(-0.5 \%)$ along this direction; whereas, the film-substrate interface along the $\mathrm{TiO}_{2}[010]$ direction is also atomically sharp, but is structurally semi-coherent, with a periodic array of misfit dislocations at the interface. These misfit dislocations are attributed to the compressive strain resulting from the $\sim 5.9 \%$ lattice mismatch along the $\mathrm{TiO}_{2}[010]$ direction. It is reasonable to associate the distorted $\vec{b}$-axis lattice constant of the film observed by $x$-ray with the semi-coherent nature of the interface along this direction observed by HREM. However, to the resolution level of the HREM studies $(-0.2 \AA)$, the majority of the strain associated with the dislocations was localized to within only a few lattice constants from the interface, in contrast to the bulk distortion observed by $x$-ray. For the purpose of the waveguide analysis, necessary rotation angle for the dielectric tensor determined from the $a$ and $c$ latice parameters yields a value of $\theta=57.16^{\circ}$, which compares with $\theta=57.215^{\circ}$ for single crystal rutile.

In order to characterize the waveguide properties of our epitaxial (101) oriented rutile thin films grown on sapphire (1120), we performed prism-film coupling experiments. In Fig. 3, we show the reflected intensity of $0.6328 \mu \mathrm{m}$ HeNe laser light from the base of $45^{\circ}$ and $60^{\circ}$ rutile 
prisms as a function of effective propagation corstant, $\beta=x_{0} n_{p} \sin \theta_{3}$ (the data from the two different prisms was combined). Three TE and one TM modes are clearly observed. In addition, two features with distorted line shapes were observed very near the substrate cutoff which seem to correspond to a possible fourth TE and second TM mode. The observed $\beta / x_{0}$ values for these features are given in Table $I$.

\begin{tabular}{ccccc}
\hline Polarization & $\mathrm{m}$ & \multicolumn{3}{c}{$\beta / \mathrm{K}_{0}$} \\
\cline { 3 - 5 } & & Osserved & Theory & Diff. \\
\hline \multirow{3}{*}{ TE } & 0 & 2.5206 & 2.5207 & -0.0001 \\
& 1 & 2.3682 & 2.3682 & +0.0000 \\
& 2 & 2.1011 & 2.1009 & +0.0002 \\
& 3 & $<1.75$ & -- & $\cdots$ \\
\hline \multirow{3}{*}{ TM } & 0 & $*$ & 2.6362 & $\cdots$ \\
& 1 & $*$ & 2.4635 &.- \\
& 2 & 2.1787 & 2.1605 & -0.0131 \\
& 3 & $<1.7771$ & 1.7815 & $>0.0044$ \\
\hline
\end{tabular}

Table I. Waveguide Results

*These modes were predicted to be outside the available $\beta / x_{0}$ range of either a $45^{\circ}$ or $60^{\circ}$ rutile prism and were not observed.
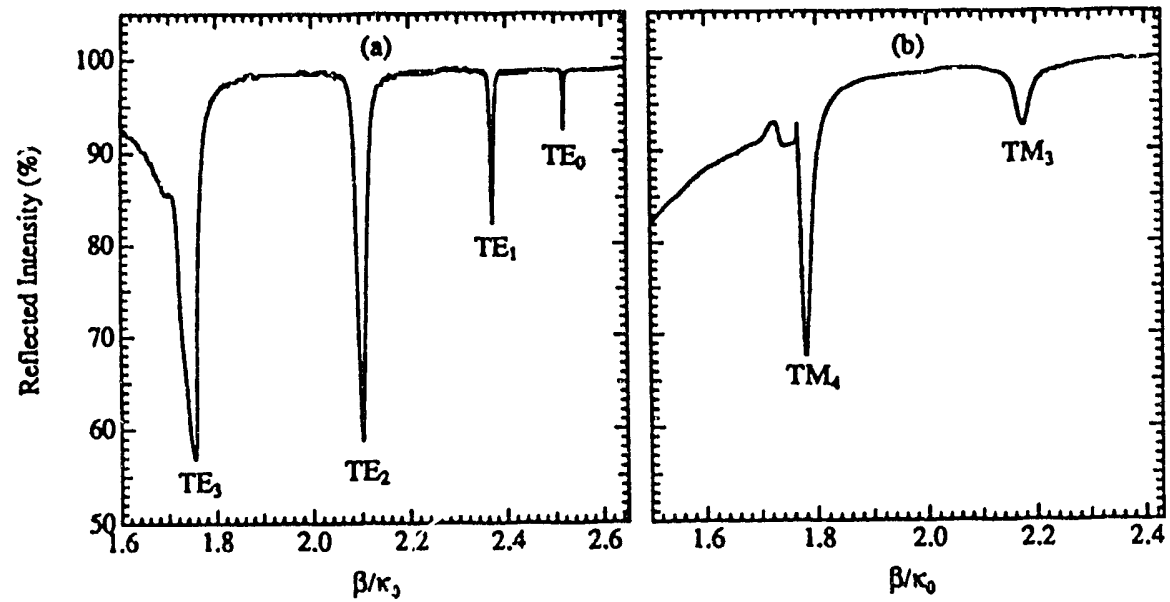

Fig. 3. Results of prism coupling experiments on epitaxial rutile (101) films on sapphire (1120). Three TE modes are shown in (a) and one TM mode is shown in (b). The modes designated $\mathrm{TE}_{3}$ and $\mathrm{TM}_{3}$ are very close to the substrate cut-off and were not used in subsequent analysis.

As shown in Ref. [10], a single equation can be obtained (for either TE or TM modes) for the film thickness as a function of the propagation constant $\beta$, with the refractive indexes of the substrate, $n_{0}$, the air gap, $n_{1}$, and the dielectric properties of the film, $K_{u}, K_{y}$ and $K_{v}$, the film thickness $2 w$, and the rotation angle $\theta$ as parameters. The equation for the TE modes is independent of $K_{u}, K_{v}$ and $\boldsymbol{\theta}$; therefore, the values of $K_{y}$ and $2 w$ may be determined independently. Since none of the films measured were of such a thickness to allow more than one TM mode to be clearly observed, $\mathbf{K}_{\mathbf{u}}$ and $\mathbf{K}_{\mathrm{v}}$ could not be determine separately. Due to a distorted line shape, the $\mathrm{TM}_{3}$ mode observed in Fig. $3 \mathrm{~b}$ was not considered in the analysis where $\mathbf{K}_{\mathrm{u}}=\mathbf{K}_{\mathrm{y}}$ was assumed. By fitting the $\beta / \boldsymbol{x}_{0}$ values observed in Fig. 3 , the best assignment of mode orders were obtained along with the following properties for the film: $\mathbf{n}_{\text {film }}^{0}=2.5701 \pm 0.0005$; $n_{\text {frim }}^{e}=2.934 \pm 0.0015$ at $\lambda=0.6328 \mu \mathrm{m}$ and a film thickness of $2 w=0.5332 \pm 0.0015 \mu \mathrm{m}$. These values of the film refractive indexes are typical of all films measured. 
The measured refractive indexes of the films are near to the properties of bulk $\mathrm{TiO}_{2}$ single crystals, indicating the high quality of these films. However, two structure features of the films should result in deviations of the dielectric properties of the films relative to bulk. First, from the $x-$ ray data, the films have a slightly mosaic structure both parallel and perpendicular to the growth plane $\left(\sim 0.3^{\circ}\right.$ and $-0.8^{\circ}$, respectively) which should result in an small averaging of the anisotropic dielectric properties of the films. Secondly, the $x$-say refinement indicated a substantial contraction of the film $\vec{b}$-axis lattice constant and a small extension of the film c-axis lattice constant relative to that of bulk rutile. Such a distorted crystal structure should be optically biaxial. To investigate this, the measured film parameters were used to determine theoretical values for $\beta / \boldsymbol{x}_{0}$ (assuming $K_{u}=K_{y}$ ) for the TE and TM modes following the analysis given above [10]. For the TE modes, the predicted $\beta / \kappa_{0}$ values for the assigned mode orders compared well with the measured results. For the TM modes, the predicted $\beta / x_{0}$ values for the $\mathrm{TM}_{0}$ and $T M_{1}$ modes were outside of the measurable range for our rutile prisms. An accurate measurement of the deviation of the $\mathrm{TM}_{3}$ mode $\beta / x_{0}$ value from theory could not be obtained due to a distorted line shape. The theoretical TM 2 mode $\beta / \kappa_{0}$ value, showed a larger deviation from experimental results than was observed for any of the TE modes. It is reasonable to conclude that the deviation of the $\mathrm{TM}_{2}$ mode angle could be attributed to strain induced biaxial characteristics associated with the observed structural distortion along the film $\vec{b}$-axis. This is currently being investigated.

In conclusion, we report optical waveguiding experiments using a prism-film coupler on $\mathrm{TiO}_{2}$ (rutile) thin films deposited using the MOCVD technique. The propagation constants for asymmetric planar waveguides composed of an anisotropic dielectric medium applicable to (101) oriented rutile thin films grown on (1120) sapphire substrates are derived. By application of this model to (101) oriented rutile thin films, we directly obtain values of the ordinary and extraordinary refractive indexes, $n_{0}$ and $n_{e}$, as well as film thickness. We obtain values of the refractive indexes $\left(n_{0}=2.5701 \pm 0.0015 ; n_{e}=2.934 \pm 0.001\right)$ near to that for bulk $\mathrm{TiO}_{2}$ single crystals indicating the exceptional quality of these films. The authors would like to thank Anthony Marturano for his assistance in the calculation. This work has been supported by the U.S. Department of Energy, Basic Energy Sciences-Materials Sciences under Contract \#W-31-109-ENG-38.

\section{REFERENCES}

1. D. Marcuse, Theory of Dielectric Optical Waveguides (Academic Press, New York, 1974); N. S. Kapany and J. J. Burke, Optical Wayequides (Academic Press, New York, 1972)

2. Y. Gao, K. L. Merkle, H. L. M. Chang, T. J. Zhang, and D. J. Lam, J. Mater. Res. 6, 2417 (1991); Y. Gao, K. L. Merkle, H. L. M. Chang, T. J. Zhang, and D. J. Lam, Phil. Mag. A 65, 1103 (1992).

3. H. You, H. L. M. Chang, R. P. Chiarello, and D. J. Lam, Mat. Res. Soc. Symp. Proc. 221, 181 (1991). H. L. M. Chang, H. You, Y. Gao, J. Guo, C. M. Foster, R. P. Chiarello, T. J. Zhang, and D. J. Lam, J. Mater. Res. 7, 2495 (1992).

4. P. K. Tien, R. Ulrich, and R. J. Martin, Appl. Phys. Lett. 17, 447 (1970); K. Tanaka and A. Odajima, Appl. Phys. Lett. 38, 481 (1981). C. Liao and G. I. Stegeman, Appl. Phys. Lett. 44, 164 (1984); S. S. Thöny and H. W. Lehmann, Appl. Phys. Lett. 61, 373 (1992).

5. P. K. Tien and R. Ulrich, J. Opt. Soc. Am. 60, 1325 (1970).

6. J. E. Midwinter, IEEE J. Quant. Elec. QE-6, 583 (1970); R. Uirich, J. Opt. Soc. Am. 60, 1337

(1970); P. K. Tien, Appl. Opt. 10, 2395 (1971); R. Ulrich and R. Torge, Appl. Opt. 12, 2901 (1973); H. Kogelnik and H. P Weber, J. Opt. Soc. Am. 64, 174 (1974).

7. D. P Gia Russo and J. H. Harris, J. Opt. Soc. Amer. 63, 138 (1973); M. O. Vassell, J. Opt. Soc. Am. 64, 166 (1974).

8. D. F. Nelson and J. McKenna, J. Appl. Phys. 38, 4057 (1967).

9. G. Leclerc and A. Yelon, Appl. Opt. 23, 2760 (1984); S. Yamamoto, K. Shibata and T. Makimoto, Opt. Comm. 31, 139 (1979); R. A. Andrews, IEEE J. Quant. Elec. QE-7, 523 (1971); IEEE J. Quant. Elec. QE-8, 27 (1972).

10. C. M. Foster, S.-K. Chan, H. L. M. Chang, R. P. Chiarello, T. J. Zhang, J. Guo, and D. J. Lam, J. Appl. Phys. 73 (10), 1 (1993).

11. J. M. Bennett and A. T. Glassman, in Handbook of Laser Science and Technology Vol, 4. Optical Materials: Part 2, Ed. by M. J. Weber, (CRC Press, Inc., Boca Raton, FL, 1988). 

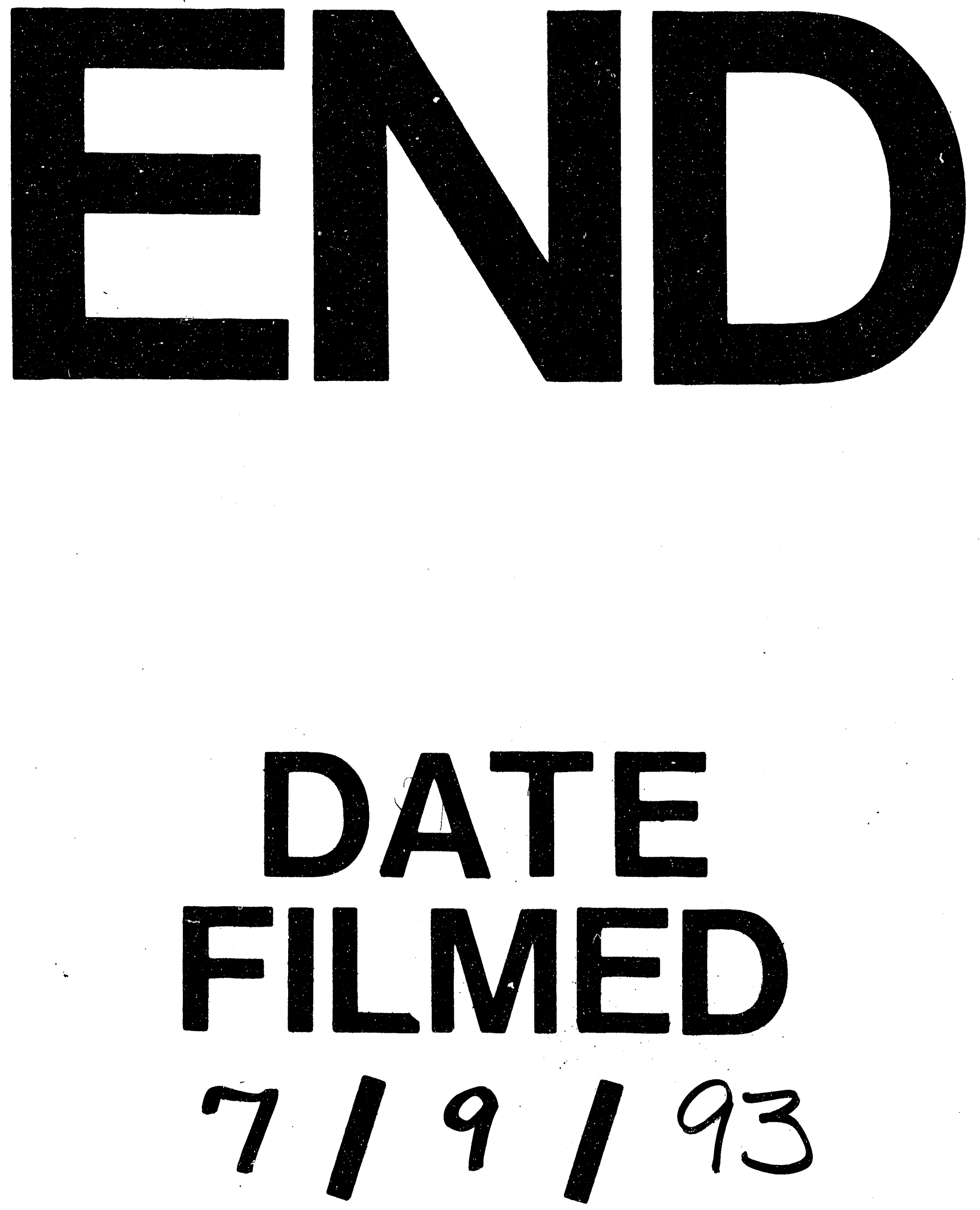
